\title{
A 1d Up Approach to Conformal Geometric Algebra: Applications in Line Fitting and Quantum Mechanics
}

\author{
Anthony N. Lasenby*®
}

\begin{abstract}
We discuss an alternative approach to the conformal geometric algebra (CGA) in which just a single extra dimension is necessary, as compared to the two normally used. This is made possible by working in a constant curvature background space, rather than the usual Euclidean space. A possible benefit, which is explored here, is that it is possible to define cost functions for geometric object matching in computer vision that are fully covariant, in particular invariant under both rotations and translations, unlike the cost functions which have been used in CGA so far. An algorithm is given for application of this method to the problem of matching sets of lines, which replaces the standard matrix singular value decomposition, by computations wholly in Geometric Algebra terms, and which may itself be of interest in more general settings. Secondly, we consider a further perhaps surprising application of the $1 \mathrm{~d}$ up approach, which is to the context of a recent paper by Joy Christian published by the Royal Society, which has made strong claims about Bell's Theorem in quantum mechanics, and its relation to the sphere $S^{7}$ and the exceptional group $E_{8}$, and proposed a new associative version of the division algebra normally thought to require the octonians. We show that what is being discussed by Christian is mathematically the same as our $1 \mathrm{~d}$ up approach to $3 \mathrm{~d}$ geometry, but that after the removal of some incorrect mathematical assertions, the results he proves in the first part of the paper, and bases the application to Bell's Theorem on, amount to no more than the statement that the combination of two rotors from the Clifford Algebra $\mathrm{Cl}(4,0)$ is also a rotor.
\end{abstract}

Mathematics Subject Classification. Primary 68-XX, 51-XX, 81-XX; Secondary 68Uxx, 81Qxx.

\footnotetext{
Sections 1 and 2 are based on part of a plenary talk given at 'AGACSE 2018: The 7th Conference on Applied Geometric Algebras in Computer Science and Engineering', July 2018, Campinas, Brazil.

This article is part of the Topical Collection on Proceedings of AGACSE 2018, IMECCUNICAMP, Campinas, Brazil, edited by Sebastià Xambó-Descamps and Carlile Lavor.
}

${ }^{*}$ Corresponding author. 
Keywords. Conformal geometric algebra, Computer vision, Quantum theory.

\section{Introduction}

For the usual CGA approach to 3d space (e.g. [6]) it is well known that we add two extra vectors. In the notation I will adopt here, these are $e$, which has $e^{2}=+1$ and $\bar{e}$, where $\bar{e}^{2}=-1$. We then define two null vectors

$$
n=e+\bar{e}, \quad \bar{n}=e-\bar{e}
$$

and use these to represent $3 \mathrm{~d}$ points $x$ using $5 \mathrm{~d}$ null vectors via

$$
X=F(x)=\frac{1}{2}\left(x^{2} n+2 x-\bar{n}\right)
$$

In this setup, Euclidean transformations of the base $3 \mathrm{~d}$ space correspond to transformations that keep the point at infinity $n$ invariant in the $5 \mathrm{~d}$ space. If instead, we look for transformations that keep $\bar{e}$ invariant, we get spherical geometry. If we look for transformations that keep $e$ invariant, we get hyperbolic geometry, as pictured in Fig. 1 in a 2d example.

It is certainly possible to use this 2d-up approach to non-Euclidean geometry, and the CGA is quite good for this, but one soon gets faced with questions like: take $X+L X L$, where $L$ is a line -it is clearly a covariant object, but what does it mean? Clearly it is no longer null, so it is not a point. One can then say using a similar approach as used for projective geometry in [7]

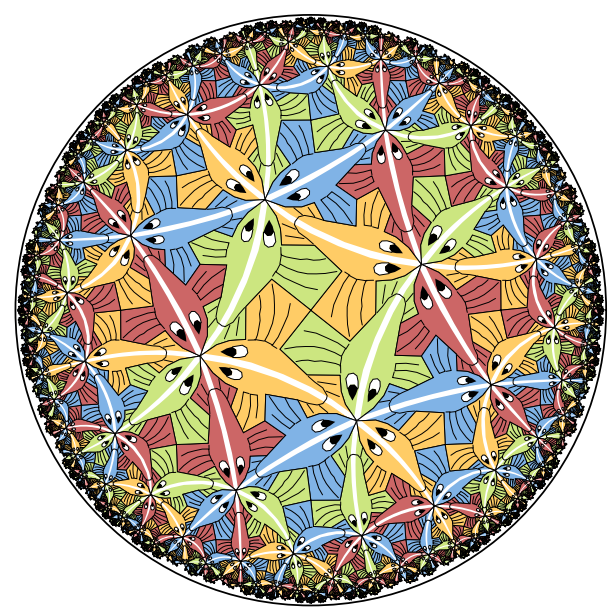

Figure 1. A rendition of Escher's circle limit III, taken from $[3]$ 


$$
Y^{\prime}=X+L X L \text { and then write } Y^{\prime}=\alpha X^{\prime}+\beta \begin{cases}e & \text { if hyperbolic } \\ \bar{e} & \text { if spherical } \\ n & \text { if Euclidean }\end{cases}
$$

to recover a new null vector $X^{\prime}$. This turns out to be a covariant operation, and so yields something geometrically meaningful. However, one quickly finds that all the extra $e$ 's or $\bar{e}$ 's or $n$ 's we have to laboriously carry around with us and then separate off, are basically irrelevant! If points don't have to be null, then we don't have to use a null vector as origin. Also hyperbolic geometry rotors do not contain $e$ (since we have to leave this invariant). E.g. the form of translation rotor is $\propto \lambda+\bar{e} a$, where $a$ is the translation vector, and $\lambda$ is a constant with dimensions 'length' which sets the curvature scale of space. Similarly for spherical rotors, there is no $\bar{e}$ in them.

Therefore, e.g. in the hyperbolic case, we can now use $\bar{e}$ as the origin, and move this around using rotors (translation and rotation) which do not contain $e$. Therefore terms in $e$ never arise this way. Similarly in the spherical case $e$ used as origin means $\bar{e}$ is never used.

Thus in both these cases, one can make do with only having one vector extra! E.g. - 3d geometry needs a 4d basis, not 5d, 4d geometry (spacetime) needs a $5 \mathrm{~d}$ basis not $6 \mathrm{~d}$. One cannot do this in the Euclidean case, since the translations there are of the form $1+(1 / 2)$ an therefore $n$ always generates both $e$ and $\bar{e}$ no matter what we take as origin.

So here is the proposal: let us do the geometry we want (even in engineering applications) in either spherical or hyperbolic space, and recover Euclidean results (if needed) by taking the limit as the length scale $\lambda \rightarrow \infty$ at the end (see below for more details of this process). In many cases this will mean 1d less for computations, and can save time in implementations.

E.g. suppose we have 4 points $a, b, c, d$ in a spherical space-how do we get the centre of a sphere passing through them? The 'old approach' would be: take $5 \mathrm{~d}$ null vector representatives $A, B, C, D$. The sphere centre is $S n S$ where $S=A \wedge B \wedge C \wedge D$. The 'new approach' is: take $4 \mathrm{~d}$ unit vector representatives $A_{i}(i=1 \cdots 4)$, and form the reciprocal frame $A^{j}$ defined by

$$
A_{i} \cdot A^{j}=\delta_{i}^{j}
$$

Now define

$$
D=\sum_{i=1}^{4} A^{i}
$$

then we find that after normalisation, $D$ is the point representing the sphere's centre and its radius can be found from $D^{2}$. This is much faster computationally. Note for more details of the 1d up approach being adopted here, see the paper [8], which has a specific focus on rigid body mechanics, but also discusses some of the geometrical issues. Further information can be found in [7]. 


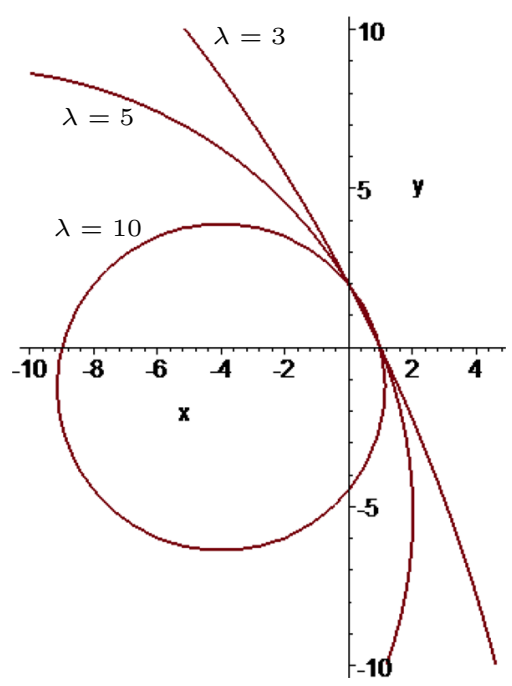

Figure 2. Lines through the fixed points $(1,0)$ and $(0,2)$, corresponding to the values 3,5 and 10 for the parameter $\lambda$

\section{Matching Groups of Lines}

We will now give a more extended example, relevant to one of the topics discussed in further papers in this collection, and which is needed in a lot of computer graphics and computer vision applications.

As an illustration, we will look at the problem of finding a common rotor (involving both translation and rotational degrees of freedom) for moving from one set of lines to a matching set. The first obvious question is how do we set up lines in the 1d-up approach? These are wedges of two 'points'. A 'point' is a unit vector (actually length -1 , since all basis vectors square to -1 in my approach, which is derived as a restriction to $3 \mathrm{~d}$ space of a spacetime metric.) Given two points we form $L=A \wedge B$ and then if $Y$ is a general point, the equation of the line is

$$
Y \wedge L=Y \wedge A \wedge B=0
$$

How this looks in our $x$-space, is then a function of the scale of the line relative to the curvature of the space, which is $4 / \lambda^{2}$. E.g., considering lines through the points $(1,0)$ and $(0,2)$, we get the lines shown in Fig. 2 for $\lambda=3$, 5 and 10 .

How do we rotate from one line to another? Suppose we have two normalised lines, $L_{1}$ and $L_{2}$ (i.e. such that $L_{1}^{2}=L_{2}^{2}=-1$ ). What we need to do is similar to what's already been described in the $2 \mathrm{~d}$-up approach in the papers by Lasenby et al. $[5,10]$. To start, we need to form the anti-commutator

$$
L_{1} L_{2}+L_{2} L_{1}=\alpha+\beta I
$$

where $I$ is the pseudoscalar for the $4 \mathrm{~d}$ space spanned by $e_{1}, e_{2}, e_{3}$ and $\bar{e}$. (Note for any bivectors $B$ and $C$ in a space of any dimension or signature, we can always write 


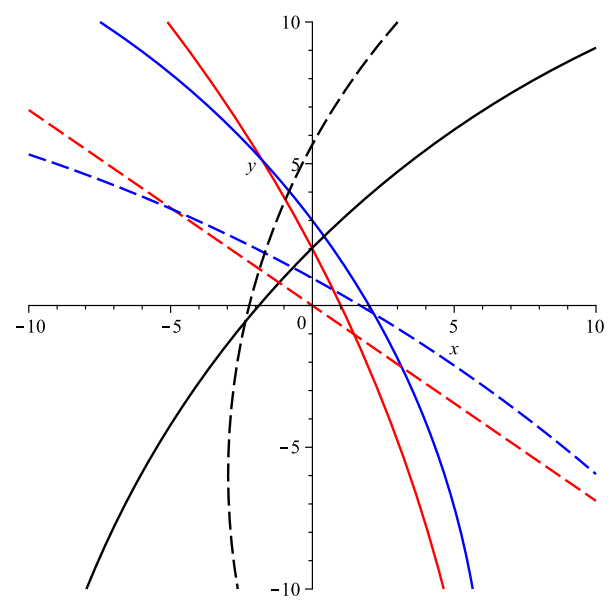

Figure 3. Two sets of lines related by a common rotation and translation. The first set are the black, red and blue lines, and the second set are 'dashed' versions of these

$$
B C=B \cdot C+B \times C+B \wedge C=\frac{1}{2}(B C+C B)+\frac{1}{2}(B C-C B)
$$

so in $4 \mathrm{~d}$, the symmetric part pulls out a scalar $(\alpha)$ and pseudoscalar part $(\beta I)$. Both of these will be invariant under all rotations in the $4 \mathrm{~d}$ space.)

Defining

$$
u=\sqrt{2-\alpha+\beta}, \quad v=\sqrt{2-\alpha-\beta}
$$

then it turns out that the following rotates from $L_{1}$ to $L_{2}$

$$
R=\frac{1}{2 u v}(u+v+(u-v) I)\left(1-L_{2} L_{1}\right)
$$

So given two lines, we can get from one to another. What about if we have a set of lines, $L_{i}, i=1 \cdots N$ and another set $L_{i}^{\prime}, i=1 \cdots N$, which are meant to be related to the first set by a common rotation and translation. An example of such sets of lines is shown in Fig. 3. As a cost function, we think the best way to proceed is to minimise

$$
S=-\sum_{i}\left\langle\left(L_{i}^{\prime}-R L_{i} \tilde{R}\right)^{2}\right\rangle_{0}
$$

with respect to varying the rotor $R$. This works, since even though $\left(L_{i}^{\prime}-R L_{i} \tilde{R}\right)^{2}$ can have a grade 4 part of either sign, its scalar part is always strictly negative, unless $L_{i}^{\prime}-R L_{i} \tilde{R}$ happens to be 0 . (Note this is a crucial difference with the $2 \mathrm{~d}$-up approach - there one can get 0 as the scalar part of a square, even if the difference is non-zero- e.g. for parallel lines - due to $n^{2}=0$.)

This problem is now very similar to the equivalent one for lines in ordinary 3d space, which Lasenby et al. looked at in 1996 [9]. Since we wish to discuss what's written in this text, we now give two short extracts. 
As an example of multivector differentiation we will consider the problem of finding the rotor $R$ which 'most closely' rotates the vectors $\left\{\boldsymbol{u}_{i}\right\}$ onto the vectors $\left\{\boldsymbol{v}_{i}\right\}, i=1, \ldots, n$. More precisely, we wish to find the rotor $R$ which minimizes

$$
\phi=\sum_{i=1}^{n}\left(\boldsymbol{v}_{i}-R \boldsymbol{u}_{i} \tilde{R}\right)^{2}
$$

Expanding $\phi$ gives

$$
\begin{aligned}
\phi & =\sum_{i=1}^{n}\left(\boldsymbol{v}_{i}^{2}-\boldsymbol{v}_{i} R \boldsymbol{u}_{i} \tilde{R}-R \boldsymbol{u}_{i} \tilde{R} \boldsymbol{v}_{i}+R\left(\boldsymbol{u}_{i}^{2}\right) \tilde{R}\right) \\
& =\sum_{i=1}^{n}\left\{\left(\boldsymbol{v}_{i}^{2}+\boldsymbol{u}_{i}^{2}\right)-2\left\langle\boldsymbol{v}_{i} R \boldsymbol{u}_{i} \tilde{R}\right\rangle\right\}
\end{aligned}
$$

To minimize $\phi$ we choose not to differentiate directly with respect to $R$ since the definition of $R$ involves the constraint $R \tilde{R}=1$, and this would have to be included via a Lagrange multiplier. Instead we use Eq. (47) to take the multivector derivative of $\phi$ with respect to $\psi$, where we replace $R \boldsymbol{u}_{i} \tilde{R}$ with $\psi \boldsymbol{u}_{i} \psi^{-1}$.

$$
\begin{aligned}
\partial_{\psi} \phi(\psi) & =-2 \sum_{i=1}^{n} \partial_{\psi}\left\langle\boldsymbol{v}_{i} \psi \boldsymbol{u}_{i} \psi^{-1}\right\rangle \\
& =-2 \sum_{i=1}^{n}\left\{\dot{\partial}_{\psi}\left\langle\dot{\psi} A_{i}\right\rangle+\dot{\partial}_{\psi}\left\langle B_{i} \psi^{-1}\right\rangle\right\}
\end{aligned}
$$

where $A_{i}=\boldsymbol{u}_{i} \psi^{-1} \boldsymbol{v}_{i}$ and $B_{i}=\boldsymbol{v}_{i} \psi \boldsymbol{u}_{i}$ (using the cyclic reordering property). The first term is easily evaluated to give $A_{i}$. To evaluate the second term we can use Eq. (47) One can then substitute $\psi=R$ and note that $R^{-1}=\tilde{R}$ as $R \tilde{R}=1$.

The crucial step here is the formula $\partial_{\psi}\left\langle M \psi^{-1}\right\rangle=\psi^{-1} P_{\psi}(M) \psi^{-1}$ which is the Eq. (47) referred to in the above extract. Then quoting again from [9], one uses this to say

$$
\begin{aligned}
\partial_{\psi} \phi(\psi) & =-2 \sum_{i=1}^{n}\left\{\boldsymbol{u}_{i} \psi^{-1} \boldsymbol{v}_{i}-\psi^{-1}\left(\boldsymbol{v}_{i} \psi \boldsymbol{u}_{i}\right) \psi^{-1}\right\} \\
& =-2 \psi^{-1} \sum_{i=1}^{n}\left\{\left(\psi \boldsymbol{u}_{i} \psi^{-1}\right) \boldsymbol{v}_{i}-\boldsymbol{v}_{i}\left(\psi \boldsymbol{u}_{i} \psi^{-1}\right)\right\} \\
& =4 \tilde{R} \sum_{i=1}^{n} \boldsymbol{v}_{i} \wedge\left(R \boldsymbol{u}_{i} \tilde{R}\right)
\end{aligned}
$$

Thus the rotor which minimizes the least-squares expression $\phi(R)=$ $\sum_{i=1}^{n}\left(\boldsymbol{v}_{i}-R \boldsymbol{u}_{i} \tilde{R}\right)^{2}$ must satisfy

$$
\sum_{i=1}^{n} \boldsymbol{v}_{i} \wedge\left(R \boldsymbol{u}_{i} \tilde{R}\right)=0
$$

This is intuitively obvious - we want the $R$ which makes $\boldsymbol{u}_{i}$ 'most parallel' to $\boldsymbol{v}_{i}$ in the average sense. The solution of Eq. (54) for $R$ will utilize 
the linear algebra framework of geometric algebra and will be described in Section 3.3.

What then happened in the IJCV paper [9], was that we manipulated this into a matrix form on which a singular value decomposition (SVD) could be employed to find $R$. Of interest here is to recast these steps into wholly GA form, and as applied to '1d-up' bivectors, rather than ordinary $3 \mathrm{~d}$ vectors. The equivalent problem here, is to find the $R$ which satisfies

$$
\sum_{i} L_{i}^{\prime} \times\left(R L_{i} \tilde{R}\right)=0
$$

where the $L_{i}$ are the set of original lines, and the $L_{i}^{\prime}$ are the 'destination' lines, for which we want to find the best rotor $R$. One soon finds this is equivalent to finding a rotor $R$ which makes the following function $g$ symmetric:

$$
g(B)=R f(B) \tilde{R}, \quad \text { and where } \quad f(B)=\sum_{i}\left(B \cdot L_{i}^{\prime}\right) L_{i}
$$

The GA way in which we can attack this is analogous to the 'polar decomposition' for an object in the conventional CGA discussed by Dorst and Valkenburg [2]. Specifically, in that approach, given an object $X$, we seek to split it up as $X=U S$ with $U$ a rotor and $S$ a 'self-reverse' element. The starting point is to form $\tilde{X} X=\tilde{S} S=S^{2}$ which we then 'square root' and can then read off $U$ as $X S^{-1}$. Analogously, here we form the function $F=\bar{g} g$, which it's easy to show is the same as $\bar{f} f$, so we can explicitly calculate it without knowing $R$. (Note for clarity we are now putting an underline on the original function, to match the overbar on the adjoint.) In fact explicitly

$$
F(B)=\sum_{i} \sum_{j}\left(B \cdot L_{j}^{\prime}\right) L_{i}^{\prime}\left(L_{i} \cdot L_{j}\right)
$$

We then calculate the eigenbivectors and eigenvalues of $F$-these are the solutions of

$$
F(B)=\lambda B
$$

and we find 4 of them here, which we label $\lambda_{k}$ and $B_{k}, k=1 \cdots 4$. We can express $F$ in terms of them as

$$
F(B)=\sum_{k} \lambda_{k}\left(B \cdot E^{k}\right) E_{k}
$$

where the $E^{k}$ are the reciprocal frame to the $E_{k}$ (this takes care of sign issues). We then note, following from the reciprocal frame properties, that

$$
F^{n}(B)=\sum_{k} \lambda_{k}^{n}\left(B \cdot E^{k}\right) E_{k}
$$

We then employ this with $n=-1 / 2$ to deduce, in the case that $g$ is symmetric, which is what we want our rotor to achieve

$$
F^{-1 / 2}(B)=(\bar{g} \underline{g})^{-1 / 2}(B)=(g g)^{-1 / 2}(B)=g^{-1}(B)=(\bar{f} \underline{f})^{-1 / 2}(B)
$$

But quite generally

$$
g^{-1}(B)=\underline{f}^{-1}(\tilde{R} B R)
$$


Hence

$$
(\bar{f} \underline{f})^{-1 / 2}(B)=\underline{f}^{-1}(\tilde{R} B R)
$$

and so, unwrapping this,

$$
R B \tilde{R}=(\bar{f} \underline{f})^{-1 / 2} \bar{f}(B)
$$

and we have succeeded in finding the action of our rotor on an arbitrary bivector.

Hopefully it will be possible to code this up soon, and test against other methods for matching line sets. As aimed for, the 'cost function' here is completely covariant and automatically decides the relative ratio of importance of rotational and translational errors. It has not yet been examined how the value of the inverse curvature scale $\lambda$ affects things - presumably it is this which tunes the relative importance of one set of errors versus the other, and it will be interesting to compare with results for matching in the $2 \mathrm{~d}$-up setting found by Joan Lasenby et al. [5].

The 2d-up setting in this latter paper is of course dealing with Euclidean rather than non-Euclidean geometry, and it may be wondered how we can make a transition to Euclidean geometry in the current approach. This was speculated about at the end of Section 18.3 in [8], where it was suggested that retaining appropriate terms in a power series expansion in $\lambda$ would be a way of achieving this. This approach has now been verified to work, and surprisingly, as suggested in [8], the appropriate terms are not the zeroth order results, but those at first order (and in some particular cases second order) in $\lambda$. This will be explained in detail elsewhere, but we show in Fig. 4. the equivalent to Fig. 3 obtained by making a first order expansion of the relevant rotors in $\lambda$. This successfully produces the equivalent setup of 'before' and 'after' lines in Euclidean space. We believe the procedure is entirely covariant, so again, it will be interesting to see how this interacts with the ' $1 \mathrm{~d}$-up' cost function, and what it means for the cost function in Euclidean space. It will also be interesting to compare with the 'Projective Geometric Algebra' approach to Euclidean space by Gunn and De Keninck [4], which also only uses 1 dimension extra.

\section{Some Comments on a Recent Paper by Joy Christian}

In 2018, a paper was published in a Royal Society journal by Christian [1], which was discussed during the AGACSE 2018 meeting in Campinas, Brazil. This paper is called 'Quantum correlations are weaved by the spinors of the Euclidean primitives', and makes some strong claims about Bell's Theorem in quantum mechanics, and its relation to the sphere $S^{7}$ and the exceptional group $E_{8}$. Perhaps most startling mathematically, as against physically, however, is the author's claim to have discovered a new associative version of the normed division algebra hitherto represented by the octonians. Joy Christian uses Geometric Algebra in his work, and claims that his new physical results in Quantum Mechanics stem from the employment of GA, and the 


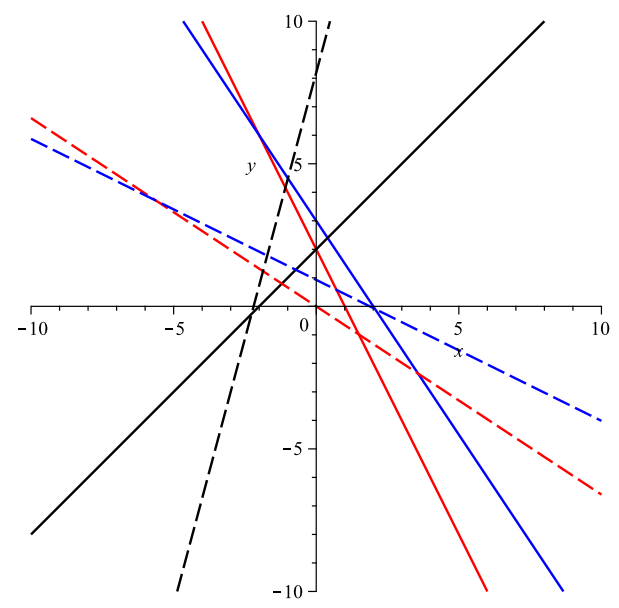

Figure 4. Same as in Fig. 3 but in the Euclidean limit obtained by a first order expansion of the rotors in the scale parameter $\lambda$

additional geometrical elements of reality which it can introduce in addition to the usual complex numbers used in Quantum Mechanics. In previous papers he has mainly considered the ordinary GA, but in the 2018 Royal Society paper he says he is considering the 'Conformal Geometric Algebra' and explicitly links the 'Euclidean primitives' of CGA with his statements about Bell's Theorem.

Christian work has repeatedly been criticised mathematically, but he has several times stated that no one well-versed in Geometric Algebra has explicitly criticised his mathematics in print, and that this suggests his critics simply do not understand the GA in his work, not that his mathematics is wrong. Stimulated by the discussion at the AGACSE meeting, I have looked at the first sections of the Royal Society paper, and found that (a), quite surprisingly, the CGA he is using is in fact a version of the 1d-up approach I have been suggesting, and (b) it is possible to find where the mathematical mistakes lie which lead to his conclusions concerning a new associative division algebra. Thus this current contribution on the 1d-up approach, provides an opportunity to record these comments as regards the mathematics that he appears to be using, and hopefully warn about the mistakes involved. However, it is very necessary to stress that this in no way is meant to transfer through to being a comment about what Christian is saying as regards Bell's Theorem. At a certain level there is certainly an interest in taking a 1d-up approach to quantum mechanics and electromagnetism, as discussed briefly by myself in a relativistic context in [7], but this area lies outside the discussion we will attempt here. In particular the comments here only relate to Sections 1 and 2 of the Christian paper, before the main work on Bell's Theorem begins. Of course the mathematical problems and misstatements in this first part of the paper would certainly need to be remedied before being able 
to approach the second part properly, hence it seems of value to record the objections here. (Note several of the points made here have been made independently by Richard D. Gill and others in the discussion thread attached to the Royal Society paper: https://royalsocietypublishing.org/doi/full/10. 1098/rsos.180526\#disqus_thread, but what may be useful here is decoding what Christian is claiming in terms of the unexpected link with the $1 \mathrm{~d}$-up approach, and also making a statement on these issues from a practicing GA person.

\subsection{Initial Problems}

(Note most equation numbers from now on relate to those in the paper [1], and we will say explicitly if we mean an equation in the current contribution.)

The first maths problem comes in Christian's Eq. (2.20), which says

$$
e_{\infty}^{2}=0
$$

and then that

Such a vector that is orthogonal to itself is called a null vector in Conformal Geometric Algebra [18]. It is introduced to represent both finite points in space as well as points at infinity [19]. As points thus defined are null-dimensional or dimensionless, addition of $e_{\infty}$ into the algebraic structure of $\mathbb{E}^{3}$ does not alter the latter's dimensions but only its point-set topology, rendering it diffeomorphic to a closed, compact, simply connected 3-sphere ...

I do not see how it can be thought introducing $e_{\infty}$ into the algebraic structure of $\mathbb{E}^{3}$ does not alter its dimensions. Also, since null vectors are described as representing both finite points as well as points at infinity, which is true in the CGA, the logic of this paragraph seems to be that since these points are null-dimensional or dimensionless then addition of any of them into $\mathbb{E}^{3}$ could go ahead and $\mathbb{E}^{3}$ would only be changed in topology.

The main problem with (2.20), however, is that it is shortly contradicted by $(2.32)$, which says that $e_{\infty}^{2}=1$. To give the full context at this point, it is said:

The three-dimensional physical space-i.e. the compact 3-sphere we discussed above - can now be viewed as embedded in the fourdimensional ambient space, $\mathbb{R}^{4}$, as depicted in Fig. 2. In this higher dimensional space, $e_{\infty}$ is then a unit vector,

$$
\left\|e_{\infty}^{2}\right\|=e_{\infty} \cdot e_{\infty}=1 \Longleftrightarrow e_{\infty}^{2}=1
$$

and the corresponding algebraic representation space (2.31) is nothing but the eight-dimensional even sub-algebra of the $2^{4}=$ 16-dimensional Clifford algebra $C l_{4,0}$. Thus, a one-dimensional subspace - represented by the unit vector $e_{\infty}$ in the ambient space $\mathbb{R}^{4}$ - represents a null-dimensional space-i.e. the infinite point of $\mathbb{E}^{3}$ - in the physical space $S^{3}$.

Again this is very difficult to understand mathematically, as anything which tries to reconcile $e_{\infty}^{2}=0$ in $3 \mathrm{~d}$, but with the same object squaring to +1 when interpreted as living in $4 \mathrm{~d}$, is bound to be. 
The next problem is Eq. (2.25), which concerns the reversion properties of the pseudoscalar $I_{c}$, which is introduced in Eq. (2.24) as

$$
I_{c}=e_{x} e_{y} e_{z} e_{\infty}
$$

The multiplication properties given for the 8 quantities

$$
\left\{1, e_{x} e_{y}, e_{z} e_{x}, e_{y} e_{z}, e_{x} e_{\infty}, e_{y} e_{\infty}, e_{z} e_{\infty}, I_{c}\right\}
$$

in Table 1, tell us unambiguously that these quantities correspond to the 8 elements of the even subalgebra of $C l_{4,0}$, with $I_{c}$ being the pseudoscalar for this space. This is not different from what Christian says, but he says in Eq. (2.25) that $I_{c}$ reverses to minus itself, i.e. (to quote)

$$
I_{c}^{\dagger}=I_{3}^{\dagger} e_{\infty}=-I_{3} e_{\infty}=-I_{c}
$$

(Note there appears to be no dispute over the dagger operation being 'reversion', and we will denote it with the usual tilde from now on.)

But this equation is wrong. We have

$$
\tilde{I}_{c}=e_{\infty} e_{z} e_{y} e_{x}=-e_{z} e_{y} e_{x} e_{\infty}=-e_{y} e_{x} e_{z} e_{\infty}=e_{x} e_{y} e_{z} e_{\infty}=I_{c}
$$

i.e. it reverses to plus itself. Thus if Christian's Eq. (2.25) is used anywhere, it will lead to error.

The next problem is with Eqs. (2.33) and (2.34), which read

$$
\begin{aligned}
& \mathcal{K}^{+}=\operatorname{span}\left\{1, e_{x} e_{y}, e_{z} e_{x}, e_{y} e_{z}, e_{x} e_{\infty}, e_{y} e_{\infty}, e_{z} e_{\infty}, I_{c}\right\} \\
& \mathcal{K}^{-}=\operatorname{span}\left\{1,-e_{x} e_{y},-e_{z} e_{x},-e_{y} e_{z},-e_{x} e_{\infty},-e_{y} e_{\infty},-e_{z} e_{\infty},-I_{c}\right\}
\end{aligned}
$$

It seems to be important to Christian's later purposes that $\mathcal{K}^{+}$and $\mathcal{K}^{-}$are different, but as spans of objects which differ just by scalar factors from the same objects in the other set, they are mathematically identical. Presumably something different is meant from what is actually written at this point, but this would have to be explained, using some concrete definitions, before the differences between $\mathcal{K}^{+}$and $\mathcal{K}^{-}$could be used later in the paper.

The next problem is with the title and initial remarks of Section 2.4 in the Christian paper. The title is 'Representation space $\mathcal{K}^{\lambda}$ remains closed under multiplication' and the initial remarks are 'As an eight-dimensional linear vector space, $K^{\lambda}$ has some remarkable properties. To begin with, $K^{\lambda}$ is closed under multiplication.' The title and remarks seem odd - we are dealing with the even subset of the Clifford algebra $C l_{4,0}$ so what is said here follows immediately from this fact. The properties are hardly remarkable per se.

More serious is what happens next. It is clear from Christian's Eq. (2.8) that by 'norm' of a general multivector $M$ he means

$$
\|M\|=\sqrt{\langle M \tilde{M}\rangle} .
$$

We can see that this square root is valid, and won't lead to imaginaries, as follows. Let us set up a general $M$ via defining two 2 -spinors $\phi$ and $\chi$ as

$$
\begin{aligned}
& \phi=a_{0}+a_{1} e_{y} e_{z}+a_{2} e_{z} e_{x}+a_{3} e_{x} e_{y} \\
& \chi=b_{0}+b_{1} e_{y} e_{z}+b_{2} e_{z} e_{x}+b_{3} e_{x} e_{y}
\end{aligned}
$$


(where the $a_{\mu}$ and $b_{\mu}, \mu=0, \ldots, 3$, are scalars) and write

$$
M=\phi+I \chi
$$

(Note we are going to write $I_{c}$ as $I$ from now on). Since $I^{2}=1$ we have

$$
M \tilde{M}=\phi \tilde{\phi}+\chi \tilde{\chi}+I(\phi \tilde{\chi}+\chi \tilde{\phi}) .
$$

Now, let us define two 4-vectors using the components of $\phi$ and $\chi$

$$
\begin{gathered}
a=a_{0} e_{\infty}+a_{1} e_{1}+a_{2} e_{2}+a_{3} e_{3} \\
b=b_{0} e_{\infty}+b_{1} e_{1}+b_{2} e_{2}+b_{3} e_{3}
\end{gathered}
$$

(Note we are not saying that $\phi$ or $\chi$ are 4 -vectors. We are just defining objects that make it easy to display the components of $M \tilde{M}$.) Then we find $\phi \tilde{\phi}+\chi \tilde{\chi}=a^{2}+b^{2}$ and $\phi \tilde{\chi}+\chi \tilde{\phi}$ is the scalar $2 a \cdot b$, meaning

$$
M \tilde{M}=a^{2}+b^{2}+2 a \cdot b I .
$$

This shows us that $\langle M \tilde{M}\rangle=a^{2}+b^{2}$ is indeed positive if $M$ is non-zero, hence the norm is well-defined.

Given any two general elements $X$ and $Y$, Christian then decides to normalise them, setting

$$
\|X\|^{2}=1, \quad\|Y\|^{2}=1 .
$$

It is not clear why we would wish to do this, but as just established, it is something we can indeed carry out for any non-zero elements.

So far, so good. However, things go very wrong with Eq. (2.40). Christian states:

We shall soon see that for vectors $X$ and $Y$ in $K^{\lambda}$ (not necessarily unit) the following relation holds:

$$
\|X Y\|=\|X\|\|Y\| \quad(2.40) .
$$

(By 'vector' Christian means what we would call 'multivector' here, as is clear from the context.) However, this is false. Consider the quantities

$$
I_{+}=\frac{1}{2}(1+I), \quad I_{-}=\frac{1}{2}(1-I) .
$$

Since $I$ squares to 1 and is its own reverse, then these satisfy the relations

$$
I_{+}^{2}=I_{+} \tilde{I}_{+}=I_{+}, \quad I_{-}^{2}=I_{-} \tilde{I}_{-}=I_{-}, \quad I_{+} I_{-}=I_{-} I_{+}=0
$$

We call such quantities 'idempotents' (since they square to themselves) and this particular pair are 'orthogonal' (since their product is zero). Now let

$$
X=\sqrt{2} I_{+}, \quad Y=\sqrt{2} I_{-}
$$

These satisfy

$$
\|X\|=1, \quad\|Y\|=1, \quad \text { but } \quad\|X Y\|=0 .
$$

This disproves the assertion in Christian's (2.40). It also means that the assertion which follows it:

One of the important observations here is that, without loss of generality, we can restrict our representation space to a set of unit vectors in $K^{\lambda}$ 
is false, since if $\|X\|$ and $\|Y\|$ are unit vectors, it does not follow that $Z=X Y$ is also a unit vector, despite what Christian says in his Eq. (2.41).

In Sect. 2.5 there is a further confusion about a quantity which when first introduced squares to 0, but then later squares to 1 . In (2.47) and (2.48) the quantity $\epsilon$, which satisfies $\epsilon^{2}=0$ is brought in to allow the definition of biquaternions, via

$$
\mathbb{Q}_{z}=\boldsymbol{q}_{r}+\boldsymbol{q}_{d} \epsilon
$$

where $\boldsymbol{q}_{r}$ and $\boldsymbol{q}_{r}$ are quaternions. In Eq. (2.51), however, $\epsilon$ is identified with $-I$, and it is stated that $\epsilon^{2}=+1$. Thus the previous reference to biquaternions is not correct. What is actually being introduced is the construction we have used above, where one can write a general element of the even subalgebra of $C l_{(4,0)}$ as

$$
M=\phi+I \chi
$$

with $\phi$ and $\chi$ as given in Eq. (3.7). We called these 2 -spinors above, but it is fine to identify them as quaternions as well. So this shows that translating the quantities introduced by Christian in this section into our notation, we have

$$
\boldsymbol{q}_{r}=\phi, \quad \boldsymbol{q}_{d}=\chi, \quad \mathbb{Q}_{z}=\boldsymbol{q}_{r}+\boldsymbol{q}_{d} \epsilon=M=\phi+I \chi
$$

(A slight problem is that since Christian says that $\epsilon$ is equal to the reverse of $I$ and he believes (wrongly) that this is $-I$, some signs will start to get out of drift as regards components of his $\boldsymbol{q}_{d}$ quaternion versus our $\chi$, but this does not seem crucial.)

Now we have so far skipped over one feature of the construction of $\mathbb{Q}_{z}$, which is that Christian wants each of $\boldsymbol{q}_{r}$ and $\boldsymbol{q}_{d}$ to be normalised, with

$$
\left\|\boldsymbol{q}_{r}\right\|=\left\|\boldsymbol{q}_{d}\right\|=\varrho
$$

where $\varrho$ is some fixed scalar. He then correctly says in Eq. (2.53) that this means

$$
\left\|\mathbb{Q}_{z}\right\|=\sqrt{2} \varrho .
$$

However, things go very wrong in the next equation. Christian says

Now the normalization of $\mathbb{Q}_{z}$ in fact necessitates that every $\boldsymbol{q}_{r}$ be orthogonal to its dual $\boldsymbol{q}_{d}$

$$
\left\|\mathbb{Q}_{z}\right\|=\sqrt{2} \varrho \Longrightarrow \boldsymbol{q}_{r} \tilde{\boldsymbol{q}}_{d}+\boldsymbol{q}_{d} \tilde{\boldsymbol{q}}_{r}=0 .
$$

This is false. The same result in the above notation [as used in our Eq. (3.11)] would be that

$$
\|a\|=\varrho \text { and }\|b\|=\varrho \Longrightarrow a \cdot b=0
$$

which is patently wrong. So can we understand why Christian believes this? Tracing through what happens in the following two equations, it is clear that the mistake is made at the point where he says that it is needed for $\mathbb{Q}_{z} \tilde{\mathbb{Q}}_{z}$ to be a scalar. If it were needed then indeed it follows that $2 a \cdot b=\boldsymbol{q}_{r} \tilde{\boldsymbol{q}}_{d}+\boldsymbol{q}_{d} \tilde{\boldsymbol{q}}_{r}$ would have to vanish, but from what has been said about $\mathbb{Q}_{z}$ so far there is no such requirement - it has just been required that the norm (as defined by 
Christian and which we examined above) has to have value $2 \varrho$. It looks as though what is happening is that Christian has temporarily forgotten that the norm is just the scalar part of the product $M \tilde{M}$, not both the scalar and grade- 4 parts. This is a very important mistake.

Of course we needed a significant mistake, since it is needed to be able to prove the (false) assertion above the product of the norms being the norm of the product. This is repeated in terms of $\mathbb{Q s}_{\mathrm{s}}$ in Eq. (2.59). The 'proof' of this amounts to the fact that if two $\mathbb{Q}$ s each individually have vanishing grade-4 part when forming $\mathbb{Q}_{z} \tilde{\mathbb{Q}}_{z}$, then the product of their norms is equal to the norm of their product. This is fine, but only applies to this special class of $\mathbb{Q}$ s, not the whole even subalgebra of $C l_{(4,0)}$, as Christian claims.

There is a lot of discussion around this part of Sect. 2.5, attempting to say that due to the relations proved for the norms, therefore he has discovered a new associative version of the normed division algebra hitherto represented by the octonians, but of course this is false, as it had to be, since the relations he is talking about only apply to a limited subset of the space, not the whole space.

\subsection{Discussion}

This completes a quick survey of the initial problems in the Christian paper, taking us through to the start of the discussion concerning quantum states. Hence this is a good time to set down what the mathematical apparatus Christian has assembled to this point actually amounts to, when stripped of the incorrect results. This can be summarised as follows.

Let us consider the even subalgebra of $C l_{(4,0)}$ and pick out the elements $R$ from this which satisfy

$$
R \tilde{R}=1
$$

i.e. we pick out the set of what are usually called rotors in this space. Then Christian's working to this point boils down to the result that if $S$ is another rotor, then the combination $S R$ is a further rotor, since it satisfies

$$
S R \widetilde{(S R)}=S R \tilde{R} \tilde{S}=1 \text {. }
$$

This is for objects which are already normalised to 1 . Slightly more generally, if we define $X=\rho_{1} R$ and $Y=\rho_{2} S$, where $\rho_{1}$ and $\rho_{2}$ scalars, then the relation

$$
\|X Y\|=\|X\|\|Y\|
$$

which is the basis for Christian's claims, is true. However, this relation does not apply to all $X$ and $Y$ in $\mathcal{K}^{\lambda}$, but only to an $X$ and $Y$ which are scaled rotors. Of course we knew it could not apply to all of $\mathcal{K}^{\lambda}$ since above we gave an explicit counterexample.

Now it is a fact that the scaled rotors, while they form a group under composition (basically multiplication from the left), they do not form a group under addition. If we add two of them with some scalar coefficients, then the resulting object when multiplied with its reverse will in general have a grade4 part, meaning it is no longer a scaled rotor. This kills off any hope that the set of such objects can form a normed division algebra, as claimed. 
We can contrast this in an unambiguous fashion with the situation which operates for $S^{3}$, and its relation to the quaternions. There, if $Q$ is a quaternion (the set of which are just the even subalgebra of $C l_{3,0}$ ), then $Q \tilde{Q}$ automatically has only a scalar part, we do not need to artificially set any other part to 0 , and so there is an automatic match to $S^{3}$. As we have seen, this same type of match does not occur for the even subalgebra of $C l_{4,0}$, since while the scalar part of $X \tilde{X}$ sets up a nice match with $S^{7}$, the extra constraint from $\langle X \tilde{X}\rangle_{4}=0$ reduces the overall dimension down to 6 , and we are working just with the rotor group.

It is necessary to state that none of this is brought out or stated in the Royal Society paper itself. There it categorically states that

$$
\|X Y\|=\|X\|\|Y\|
$$

applies to all of the even subalgebra of $C l_{4,0}$, which if true would make it a genuine normed division algebra, but of course we have seen that this is mistaken.

Thus Sects. 1 and 2 of the paper succeed only in showing that the set of rotors of $C l_{4,0}$ (i.e. even elements of $C l_{4,0}$ which satisfy $M \tilde{M}=1$ ) are closed under multiplication. This is a trivial result which can be established in only a few lines. All the associated statements about normed division algebras, oriented bases, Hopf fibrations, $S^{7}, E_{8}$ etc. appear to be irrelevant and not substantiated by what is shown in the paper. Moreover, the paper itself gives no inkling that the restriction to rotors is being applied and indeed stresses the applicability of crucial formulae, such as (2.40), to all members of $C l_{4,0}$, which is unfortunately false.

It is of course possible that all Christian needs in the second part of the paper, beginning Sect. 3, is the reduced result concerning scaled rotors that has just been described, but this would need a separate development with some quite different mathematics than actually given in the paper.

Open Access. This article is licensed under a Creative Commons Attribution 4.0 International License, which permits use, sharing, adaptation, distribution and reproduction in any medium or format, as long as you give appropriate credit to the original author(s) and the source, provide a link to the Creative Commons licence, and indicate if changes were made. The images or other third party material in this article are included in the article's Creative Commons licence, unless indicated otherwise in a credit line to the material. If material is not included in the article's Creative Commons licence and your intended use is not permitted by statutory regulation or exceeds the permitted use, you will need to obtain permission directly from the copyright holder. To view a copy of this licence, visit http:// creativecommons.org/licenses/by/4.0/.

Publisher's Note Springer Nature remains neutral with regard to jurisdictional claims in published maps and institutional affiliations. 


\title{
References
}

[1] Christian, J.: Quantum correlations are weaved by the spinors of the Euclidean primitives. R. Soc. Open Sci. 5, 180526 (2018)

[2] Dorst, L., Valkenburg, R.: Square root and logarithm of rotors in 3d conformal geometric algebra using polar decomposition. In: Guide to Geometric Algebra in Practice, pp. 81-104. Springer (2011)

[3] Dunham, D.: Transformation of hyperbolic Escher patterns. Visual Math. 1, (1999)

[4] Gunn, C.G., De Keninck, S.: Geometric algebra and computer graphics. In: ACM SIGGRAPH 2019 Courses, pp. 1-140 (2019)

[5] Hadfield, H., Lasenby, J., Ramage, M., Doran, C.: REFORM: rotor estimation from object resampling and matching. Adv. Appl. Clifford Algebras 29, 67 (2019)

[6] Hestenes, D.: Old wine in new bottles: a new algebraic framework for computational geometry. In: Bayro-Corrochano, E., Sobczyk, G. (eds.) Geometric Algebra with Applications in Science and Engineering, pp. 3-17. Springer (2001)

[7] Lasenby, A.: Recent applications of conformal geometric algebra. In: Computer Algebra and Geometric Algebra with Applications, pp. 298-328. Springer (2004)

[8] Lasenby, A.: Rigid body dynamics in a constant curvature space and the ' $1 \mathrm{D}$ up' approach to conformal geometric algebra. In: Guide to Geometric Algebra in Practice, pp. 371-389. Springer (2011)

[9] Lasenby, J., Fitzgerald, W.J., Lasenby, A.N., Doran, C.: New geometric methods for computer vision: an application to structure and motion estimation. Int. J. Comput. Vis. 26, 191 (1998)

[10] Lasenby, J., Hadfield, H., Lasenby, A.: Calculating the rotor between conformal objects. Adv. Appl. Clifford Algebras 29, 102 (2019)

\author{
Anthony N. Lasenby \\ Kavli Institute for Cosmology \\ c/o Institute of Astronomy \\ Madingley Road \\ Cambridge CB3 0HA \\ UK \\ e-mail: a.n.lasenby@mrao.cam.ac.uk \\ and
}

Astrophysics Group

Cavendish Laboratory

JJ Thomson Avenue

Cambridge CB3 0HE

UK

Received: February 28, 2019.

Accepted: February 3, 2020. 